\title{
A retrospective study of six patients with mandibular metastatic carcinoma
}

\author{
ZHEN CAI $^{1 *}$, CHAO ZHU $^{1,2^{*}}$, LIZHEN WANG $^{3}$, LING ZHU $^{4}$, ZHIYUAN ZHANG $^{2}$, \\ HANGUANG ZHU ${ }^{2}$ and YAN'AN WANG ${ }^{2}$ \\ ${ }^{1}$ Department of Oral and Maxillofacial Surgery, Linyi People's Hospital, Linyi, Shandong 276003; \\ Departments of ${ }^{2}$ Oral and Maxillofacial Surgery, ${ }^{3}$ Oral Pathology and ${ }^{4}$ Radiology, Ninth People's Hospital, \\ Shanghai Jiao Tong University School of Medicine, Shanghai 200011, P.R. China
}

Received December 20, 2014; Accepted January 22, 2016

DOI: $10.3892 / \mathrm{ol} .2016 .4484$

\begin{abstract}
Mandibular metastatic carcinoma is a rare lesion that accounts for $<1 \%$ of all oral malignancies. To provide greater experience in this field, the present study was conducted in which 6 cases of mandibular metastatic carcinoma were retrospectively reviewed. The origin of the lesions was the prostate in 2 cases, the lungs in 2 cases, the breast in 1 case and the thyroid gland in 1 case. The clinical and computed tomography features, surgical management and follow-up outcomes were investigated. The study indicated that surgeons should include the suspicion of metastasis in the differential diagnosis for mandibular tumor, particularly in patients who have a history of malignancy. A poor prognosis was associated with the examined patients. To extend the survival time as long as possible, a treatment strategy using multiple therapies, including segmental mandibulectomy, radiotherapy and chemotherapy, is recommended.
\end{abstract}

\section{Introduction}

Metastatic carcinoma of the mandible is a rare lesion that constitutes $<1 \%$ of all oral malignancies (1). The primary tumors from which metastases occur most commonly arise in the breasts, lungs and prostate (1-3), followed by the kidney, thyroid gland, liver, stomach, intestines, testes and bladder (1,4-8). Surgeons often have little experience with mandibular metastatic carcinomas, hence, the majority of such tumors are mistakenly considered to be new primary growths until histological examination reveals their true metastatic

Correspondence to: Dr Yan'an Wang, Department of Oral and Maxillofacial Surgery, Ninth People's Hospital, Shanghai Jiao Tong University School of Medicine, 639 Zhi-Zao-Ju Road, Shanghai 200011, P.R. China

E-mail: wangyanan9h@163.com

*Contributed equally

Key words: mandibular metastatic carcinoma, computed tomography, differential diagnoses, therapy, survival nature $(1,2)$. There are no clear differences in histological findings between metastatic and primary lesions, and the diagnosis of mandibular metastatic carcinoma is primarily based on biopsy rather than radiographical or physical examination $(2,3)$. Usually, segmental mandibulectomy surgery, radiotherapy and chemotherapy are used to extend the survival time of patients, although a significant extension of survival time has yet to be achieved (9). However, a poor prognosis is associated with these patients $(2,3,9)$. In an early study conducted by Clausen and Poulsen (3), 70 patients were observed following the recognition of the jaw metastasis; in total, 49 of these patients succumbed to the disease within one year, 6 within 2 years and 2 within 4 years.

In order to provide a greater experience of this condition, the present retrospective study was conduced to investigate the clinical and computed tomography (CT) features of 6 patients with mandibular metastatic carcinomas, including the surgical management options and follow-up outcomes.

\section{Materials and methods}

Patients. The medical records of 6 cases of mandibular metastatic carcinoma obtained between October 17, 2002, and October 24, 2009 from the Ninth People's Hospital, Shanghai Jiao Tong University School of Medicine (Shanghai, China), were retrospectively reviewed. Only cases that reported the mandible site and had histological confirmation were included in the present study. The cases consisted of 4 women and 2 men, with an average age of 66.3 years (range, 51-81 years). The origin of the lesions was the prostate in 2 cases, the lungs in 2 cases, the breast in 1 case and the thyroid gland in 1 case (Table I).

Ethical approval was obtained from the Ethics Committee of Ninth People's Hospital, Shanghai Jiao Tong University School of Medicine [approval no., 2015(98)].

Treatment. Clinical examinations and CT scans were performed in all 6 patients pre-operatively. With the exception of case 4, all patients were treated with a segmental mandibulectomy and post-operative chemoradiotherapy. The patient in case 4 was reluctant to undergo a mandibulectomy, thus conservative enucleation was used. 


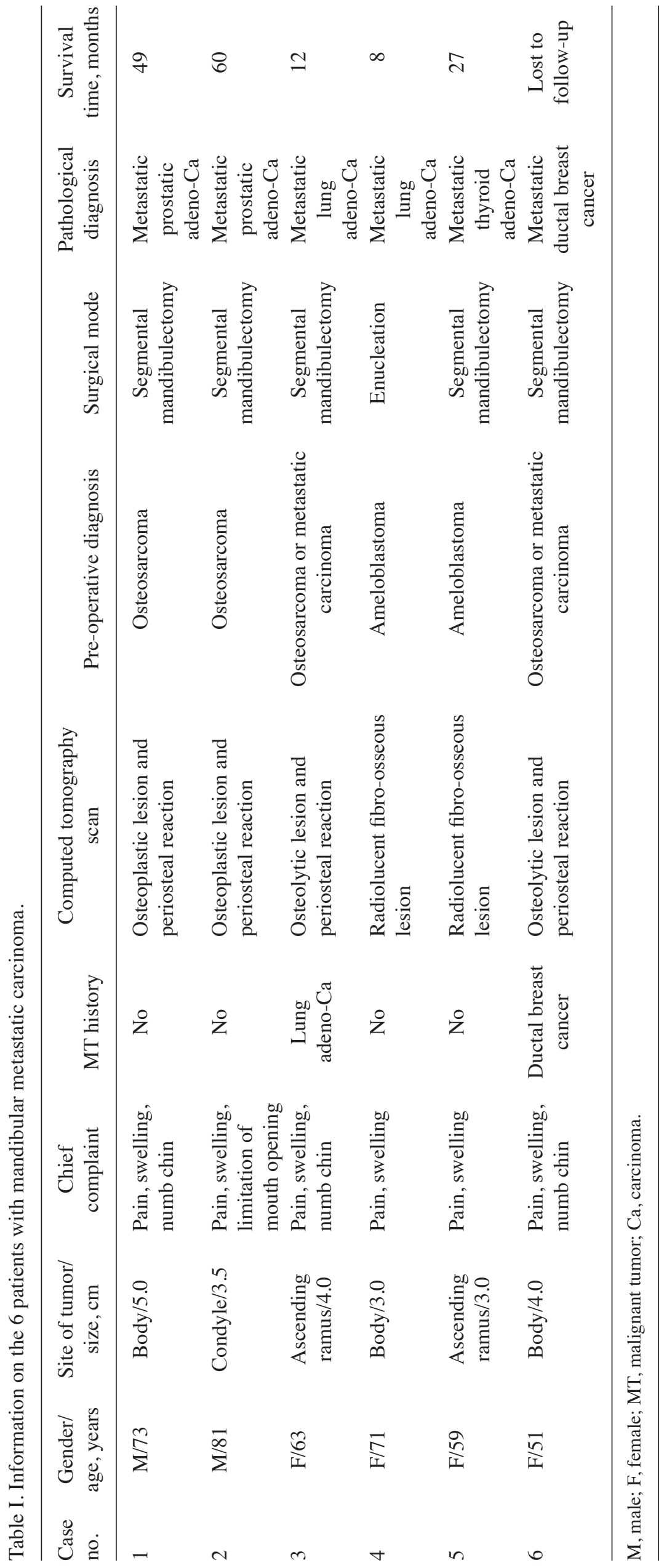




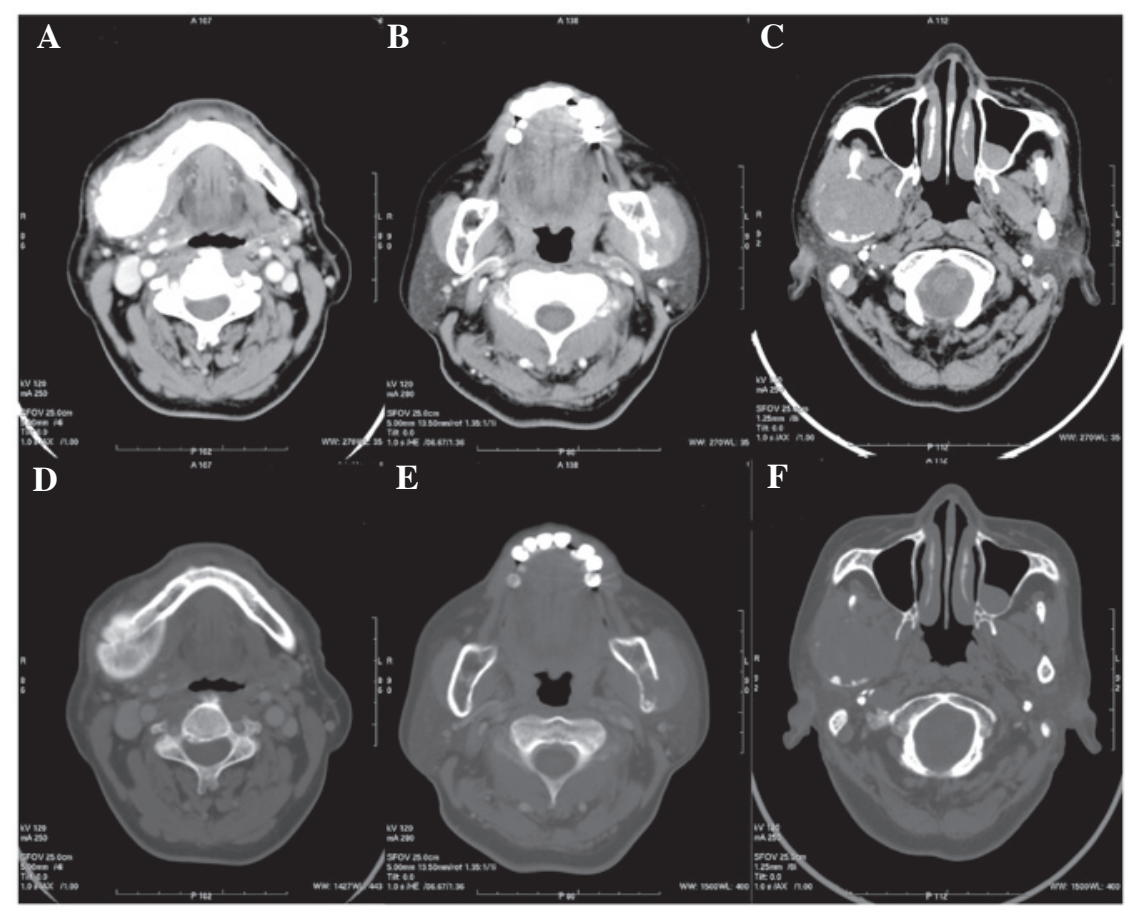

Figure 1. Computed tomography features of mandibular metastatic carcinoma. (A and D) Case 1: An osteoplastic lesion with a clear periosteal reaction; (B and E) case 3: An osteolytic lesion with a periosteal reaction; and ( $\mathrm{C}$ and F) case 5: A radiolucent fibro-osseous lesion. (A, B and C show soft tissue windows, with a window width of $270 \mathrm{Hu}$ and a window level of $35 \mathrm{Hu}$; D, E and F show bone windows, with a window width of 1,500 Hu and a window level of $400 \mathrm{Hu}$ ).

Diagnosis and follow-up. The post-operative pathological diagnoses were formed using histological images and immunohistochemistry. The post-operative follow-up period ranged from 8 to 60 months (mean, 31.2 months), which reflected the post-operative survival time.

\section{Results}

All 6 patients presented with mandibular pain and bone swelling, 3 patients experienced a numb chin and 1 patient presented with a limited degree of mouth opening. The patient in case 3 had a history of lung adenocarcinoma, while case 6 had a history of ductal breast cancer; each patient were surgically treated 1.5 years prior to the current admission. Prior to admission, the suspicion of primary recurrence was eliminated of the two patients by examination with regard to the relevant discipline. The remaining 4 patients had no history of malignant tumors.

CT scans (Fig. 1) showed that cases 1 and 2 exhibited osteoplastic lesions (Fig. 1A), while cases 3 and 6 presented with osteolytic masses (Fig. 1B); all 4 of these patients presented with the apparent radiographic feature of a periosteal reaction, which was suggestive of osteosarcoma (Fig. 1D and E). The tumors of the other 2 patients (cases 4 and 5) appeared as expansive and radiolucent masses, with the characteristics of fibro-osseous lesions (Fig. 1C and F).

The pre-operative first diagnosis of the patients in cases 1 , 2, 3 and 6 was mandibular osteosarcoma. For cases 3 and 6, considering their malignant history, metastatic carcinoma was formed as the second diagnosis following pre-operative CT. The other 2 patients (cases 4 and 5) were diagnosed with ameloblastoma mainly based on the $\mathrm{CT}$ characteristics.

In total, 5 of the patients (cases 1,2,3,5 and 6) were surgically treated with an en bloc segmental mandibulectomy with a margin of normal bone, and subsequent adjuvant therapy consisting of radiotherapy and chemotherapy. For case 4, following the enucleation of the lesion, an intraoperative rapid-frozen biopsy provided the diagnosis of malignancy. However, a further extensive osteotomy was refused by the patient's family. Alternative chemoradiotherapy was selected instead. All patients were advised to visit the relevant respective departments of the hospital for further treatment of the primary site following discharge from the Department of Oral and Maxillofacial Surgery.

The pathological diagnosis of all 6 patients was metastatic carcinoma. Cases 1 and 2 originated from the prostate (Fig. 2A), with immunostaining of prostate-specific antigen in the atypical cells confirming the diagnosis of metastatic prostatic adenocarcinoma (Fig. 3A). Cases 3 and 4 originated from lung adenocarcinoma; adenoid structures and columnar epithelial cells with cellular nucleus division were observed (Fig. 2B). Case 5 originated from thyroid adenocarcinoma (Fig. 2C), with immunostaining testing positive for thyroglobulin in the cytoplasm of the nests of tumor tissues (Fig. 3B). Case 6 originated from breast ductal cancer; a large number of ductal carcinoma cell nests were surrounded by osseous trabecular structures (Fig. 2D).

Patients from cases 1, 3, 4 and 5 succumbed to cachexia caused by cancer recurrence or extensive metastasis during the follow-up period. The patient from case 2 succumbed due to cardiac disease 60 months after surgery, and case 6 was lost to follow-up, thus a survival time could not be acquired.

\section{Discussion}

In total, $<1 \%$ of mandibular malignancies are metastatic lesions of a primary tumor originating in another region of the 

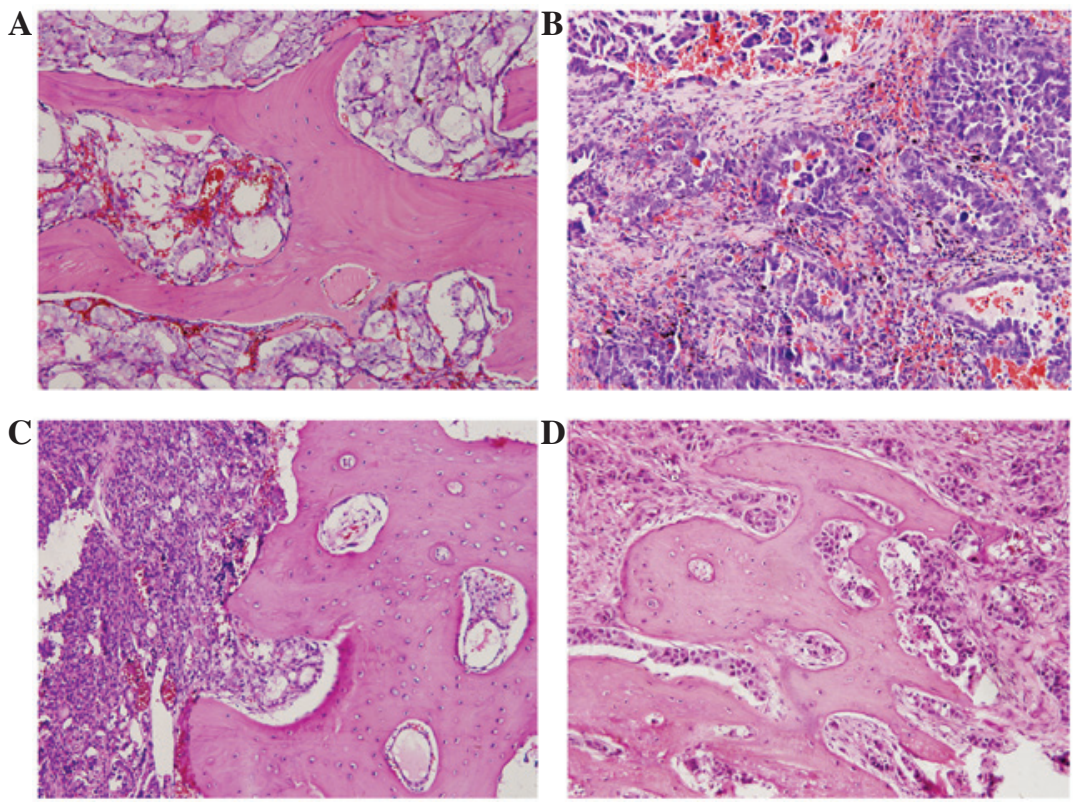

Figure 2. Histological images. (A) Case 1: A mandibular metastatic prostatic adenocarcinoma, with crushed adenocarcinoma cells found within the bone; (B) case 3: A mandibular metastatic lung adenocarcinoma, in which adenoid structures and columnar epithelial cells with cellular nucleus division was observed; (C) case 5: A mandibular metastatic thyroid adenocarcinoma, with tumor cells arranged in nests and growing within the bone; and (D) case 6: Mandibular metastatic ductal breast cancer, with ductal carcinoma cell nests surrounded by tumor connective tissue within the bone. Original magnification, $\mathrm{x} 200$.
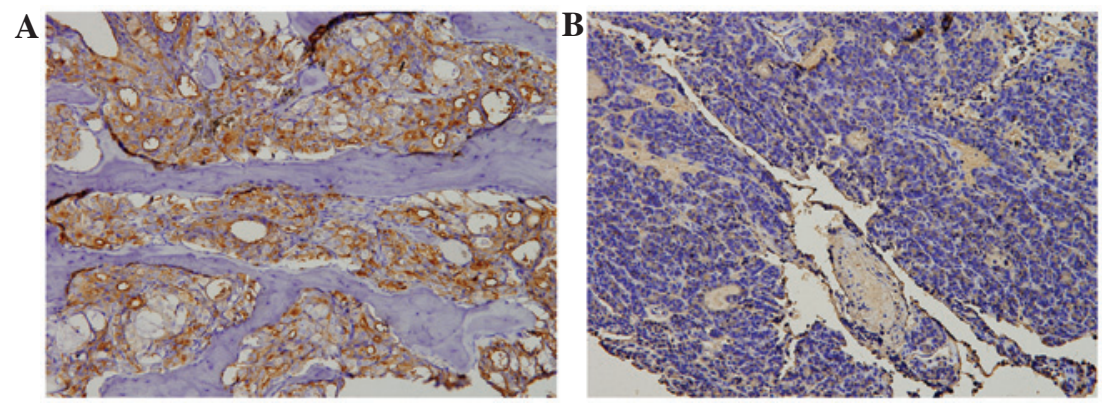

Figure 3. Immunostaining. (A) Case 1: The overexpression of prostate-specific antigen in the atypical cells of the prostatic metastatic adenocarcinoma specimen; and (B) case 5, immunostaining of thyroglobulin in the atypical cells confirming the diagnosis of thyroid metastatic adenocarcinoma. Magnification, $\mathrm{x} 200$.

body $(1,2)$. However, the actual morbidity rate may be even higher for the following reasons: i) Radiographic surveys of the jaws are not routine; ii) certain precancerous lesions and preinvasive carcinomas are hard to detect by radiographic examination; and iii) the jaws are seldom examined during autopsy (10-13).

Cases of primary carcinoma metastasizing to the mandible were previously reviewed in a study by D'Silva et al, which recorded the following primary sites: Breasts (25\%), lungs $(13 \%)$, prostate $(10 \%)$, colon $(7 \%)$, thyroid gland $(3 \%)$ and kidneys (3\%) (1). Metastasis of maxillofacial bone carcinoma to distant regions occurs primarily via the blood (2). However, there is a much higher frequency of metastasis development in the mandible compared with other maxillofacial bones $(3,10)$. The exact reason for this is unknown. The mandible is fed by the terminal inferior alveolar artery, which is tightly encased by the annular mandibular marrow cavity (14). Furthermore, the pathway of the inferior alveolar artery traveling through the mandible is quite long. We speculate that these anatomical features are more suitable for the stagnation and gathering of tumor cells, and that the nutrient-rich red active marrow within the mandible is eligible for tumor proliferation.
In the present study, pain and swelling were the most common symptoms of the mandibular malignancies, but the most significant symptom was paresthesia of the chin. Nerve numbness has a close association with malignant tumors $(3,15)$. Limitation of mouth opening was also found in the patient that presented with a condylar metastasis (case 2) in the present study. It should be noted that condylar metastasis with symptoms of temporomandibular joint pain and limited mouth opening could mimic temporomandibular disorders (TMD) (16-19). In this case, clinicians should be aware of the differential diagnosis of this tumor, particularly when patients have a history of malignancy or respond to TMD-therapy inappropriately (19).

In general, the primary tumor is diagnosed prior to the appearance of oral metastasis. However, in the present study, 4 out of the 6 cases experienced oral symptoms prior to diagnosis of the primary site. Furthermore, in certain cases, the primary malignancy remains undetected even though metastatic tumors of the jaw have been pathologically diagnosed (1).

$\mathrm{CT}$ is the conventional diagnostic tool for mandibular metastatic lesions (6). In the current study, the 2 prostatic 
metastatic cases presented with osteoplastic lesions, while others showed osteolytic change. A 'sunburst' periosteal reaction was observed in 4 lesions and the other 2 lesions showed a well-demarcated radiolucent fibro-osseous lesion. However, the diagnosis of mandibular metastatic carcinoma can be difficult to make based solely on CT features. For cases 3 and 6 , due to the history of malignancy, the possibility of metastatic carcinoma was considered in the differential diagnosis, however, it was thought more likely that the lesion was a primary osteosarcoma according to the CT results.

The differential diagnosis for mandibular metastatic carcinoma includes TMD, ameloblastoma, primary intraosseous squamous carcinoma, and particularly, osteosarcoma $(2,16,17,20,21)$. The past medical history, symptoms, clinical examination, and CT or magnetic resonance imaging (MRI) results of a patient should be carefully investigated. For patients with a history of malignancy, metastatic carcinoma must be considered in the differential diagnosis. As CT or MRI scans can provide uncharacteristic findings, biopsy is an essential and indispensable tool for forming a diagnosis (3). Additionally, if surgeons suspect that there is a possibility of metastases, then positron emission tomography (PET)/CT maybe required (22). PET/CT is usually an effective diagnostic tool for metastatic carcinoma, with the shortcoming of this equipment being its low popularity in developing regions.

Mandibular metastatic carcinoma is associated with a poor prognosis. The majority of patients diagnosed with oral metastasis succumb to the disease within 1 year, while the 4-year survival rate is estimated to be $10 \%(3,23)$. The main purpose of therapy is to relieve pain and extend the survival time. In the present study a collaborative therapeutic schedule that included surgical, chemical and radioactive treatment was recommended. Following this integrated therapy, and combined with androgen deprivation, the 2 patients in the prostatic metastatic cases achieved survival times of $>4$ years. By contrast, the patient in case 4 who rejected the extensive osteotomy succumbed to cachexia 8 months later. It should be noted that if extensive metastases have been detected pre-operatively or if the patient is in a bad general condition, palliative care may be the sensible choice.

As the general prognosis of such patients is rather poor, intraoperative immediate mandible reconstruction by bone transplantation, including fibular or iliac bone flaps, should be avoided to alleviate the risks and repercussions associated with surgery.

In conclusion, the diagnosis of mandibular metastatic carcinoma is rather difficult, particularly for patients who develop oral symptoms prior to the diagnosis of the primary site and in whom there is no history of malignancy. A poor prognosis is associated with these patients. To extend the survival time of such patients, a treatment strategy using multiple therapies, including segmental mandibulectomy, radiotherapy and chemotherapy, is recommended.

\section{References}

1. D'Silva NJ, Summerlin DJ, Cordell KG, Abdelsayed RA, Tomich CE, Hanks CT, Fear D and Meyrowitz S: Metastatic tumors in the jaws: A retrospective study of 114 cases. J Am Dent Assoc 137: 1667-1672, 2006.
2. Bigelow NH and Walsh TS: Metastatic carcinoma of the mandible. Ann Surg 137: 138-140, 1953.

3. Clausen F and Poulsen H: Metastatic carcinoma to the Jaws. Acta Pathol Microbiol Scand 57: 361-374, 1963.

4. Jones GM, Telfer MR and Eveson JW: Metastatic renal clear cell carcinoma of the jaws. Two cases illustrating clinical and pathological diagnostic problems. Br J Oral Maxillofac Surg 28: 172-175, 1990.

5. Ismail SB, Abraham MT, Zaini ZB, et al: Metastatic follicular thyroid carcinoma to the mandible: A case report. Cases J 2: 6533,2009

6. Kamatani T, Tatemoto Y, Tateishi Y and Yamamoto T: Isolated metastasis from hepatocellular carcinoma to the mandibular condyle with no evidence of any other metastases: A case report. Br J Oral Maxillofac Surg 46: 499-501, 2008.

7. Uchiyama Y, Murakami S, Kakimoto N, Nakatani A, Kishino M, Hamab Y and Furukawa S: Diagnostic imaging findings for mandibular metastasis from gastric adenocarcinoma. Oral Surg Oral Med Oral Pathol Oral Radiol Endod 107: e49-e53, 2009.

8. Babu KG, Raud C, Kumaraswamy SV and Lalitha N: Carcinoma colon with mandible and liver metastases. Br J Oral Maxillofac Surg 34: 133-134, 1996.

9. Nishikawa H, Nakashiro K, Sumida T, Sugita A and Hamakawa H: Mandibular osteoblastic metastasis of poorly differentiated carcinoma of the thyroid gland. Int J Oral Maxillofac Surg 39: 301-304, 2010

10. Freudlsperger C, Kurth R, Werner MK, Hoffmann J and Reinert S: Condylar metastasis from prostatic carcinoma mimicking temporomandibular disorder: A case report. Oral Maxillofac Surg 16: 79-82, 2012.

11. Buchner A and Ramon Y: Distant metastases to the jaws: Report of four cases. J Oral Surg 25: 246-250, 1967.

12. Cash CD, Royer RQ and Dahlin DC: Metastatic tumors of the jaws. Oral Surg Oral Med Oral Pathol 14: 897-905, 1961.

13. Neville BW and Day TA: Oral cancer and precancerous lesions. CA Cancer J Clin 52: 195-215, 2002.

14. Kim ST, Hu KS, Song WC, Kang MK, Park HD and Kim HJ: Location of the mandibular canal and the topography of its neurovascular structures. J Craniofac Surg 20: 936-939, 2009.

15. Orhan K, Bayndr H, Aksoy S, Seker BK, Berberoğlu A and Ozan O: Numb chin syndrome as a manifestation of possible breast cancer metastasis around dental implants. J Craniofac Surg 22: 942-945, 2011.

16. Tabib R, Elias S, Tal Y, Ben-Yehuda A and Abu-Tair J: Temporomandibular joint-related symptoms as initial presentation of lung carcinoma in a patient with Takayasu's arteritis. J Oral Maxillofac Surg 69: 226-229, 2011.

17. Qiu YT, Yang C, Chen MJ and Qiu Wl: Metastatic spread to the mandibular condyle as initial clinical presentation: Radiographic diagnosis and surgical experience. J Oral Maxillofac Surg 71: 809-820, 2013.

18. Kelles M, Akarcay M, Kizilay A and Samdanci E: Metastatic renal cell carcinoma to the condyle of the mandible. J Craniofac Surg 23: e302-e303, 2012.

19. Kruse AL, Luebbers HT, Obwegeser JA, Edelmann L and Graetz KW: Temporomandibular disorders associated with metastases to the temporomandibular joint: A review of the literature and 3 additional cases. Oral Surg Oral Med Oral Pathol Oral Radiol Endod 110: e21-e28, 2010.

20. Tchan MC, George M and Thomas M: Metastatic prostate cancer mimicking primary osteosarcoma of the jaw: An infrequent clinical case. South Med J 101: 657-659, 2008.

21. Yagan R, Bellon EM and Radivoyevitch M: Breast carcinoma metastatic to the mandible mimicking ameloblastoma. Oral Surg Oral Med Oral Pathol 57: 189-194, 1984.

22. Cheung PK, Chin RY and Eslick GD: Detecting residual/recurrent head neck squamous cell carcinomas using PET or PET/CT: Systematic review and meta-analysis. Otolaryngol Head Neck Surg Dec 29, 2015 (Epub ahead of print).

23. Kesting MR, Loeffelbein DJ, Hölzle F, Wolff KD and Ebsen M: Male breast cancer metastasis presenting as submandibular swelling. Auris Nasus Larynx 33: 483-485, 2006. 